\title{
Energetic BEM for the numerical analysis of 2D Dirichlet damped wave propagation exterior problems
}

\author{
A. Aimi $^{1}$, M. Diligenti ${ }^{1}$, C. Guardasoni ${ }^{1 *}$ \\ ${ }^{1}$ Department of Mathematics and Computer Science, University of Parma, Italy \\ *Email address for correspondence: chiara.guardasoni@unipr.it \\ Communicated by Luca Formaggia \\ Received on 11 07, 2016. Accepted on 05 02, 2017.
}

\begin{abstract}
Time-dependent problems modeled by hyperbolic partial differential equations can be reformulated in terms of boundary integral equations and solved via the boundary element method. In this context, the analysis of damping phenomena that occur in many physics and engineering problems is a novelty. Starting from a recently developed energetic space-time weak formulation for 1D damped wave propagation problems rewritten in terms of boundary integral equations, we develop here an extension of the so-called energetic boundary element method for the 2D case. Several numerical benchmarks, whose numerical results confirm accuracy and stability of the proposed technique, already proved for the numerical treatment of undamped wave propagation problems in several dimensions and for the 1D damped case, are illustrated and discussed.
\end{abstract}

Keywords: Damped wave equation, energy, boundary element method

AMS subject classification: 65N38

\section{Introduction}

A variety of engineering and physical applications, such as the propagation or the scattering of acoustic or electromagnetic waves, leads to the problem of solving linear hyperbolic partial differential equations (PDEs) in two or three dimensional space [1-3]. These problems are normally considered in an unbounded homogeneous domain and a method to tackle them is to reformulate the PDE as a boundary integral equation (BIE) on the usually bounded boundary of the domain, which can then be numerically solved using the boundary element method (BEM) $[4,5]$. This approach is useful especially for solving problems of practical importance with irregular geometries. Further, in some applications, the physically relevant data are given not by the solution in the interior of the domain but rather by the boundary values of the solution or its derivatives. These data can be 
A.Aimi et al.

obtained directly from the solution of BIEs, whereas it is well known that boundary values obtained with low order finite element method (FEM) solutions are in general not so accurate. Sometimes, however, BEM-FEM coupling proves to be highly useful [6-8].

In the context of wave propagation, while the elastic forces tends to maintain the oscillatory motion, the transient effect dies out because of energy dissipations. The process of energy dissipation is generally referred to as damping. The analysis of damping phenomena that occur, for example, in fluid dynamics, in kinetic theory and in semiconductors, is of particular interest [9-13]: the dissipation is generated by the interaction between the waves and the propagation medium and can be also closely related to the dispersion, as in the interactions between water streams and surface waves or in ferromagnetic materials. On the other side, in mechanical systems, in general, damping has the effect of reducing the amplitude of vibration and, therefore, it is desirable to have some amount of damping in order to faster achieve stability. Hence, damping is whether an unavoidable presence in physical reality or a desired characteristic in design.

The use of advanced numerical techniques to solve the related PDEs, such as FEM and the finite difference methods (FDM) is well established and it is standard in this framework, even if the research of a numerical method that could reproduce the expected damping decay is an actual argument in literature (see [14-16] and their references). On the other hand, in the context of BEMs the analysis of dissipation through damped wave equation rewritten as a BIE is a relatively new topic, because it has been scarcely investigated until now. Since wave propagation phenomena are often observed in semi-infinite media (domain) where Sommerfeld radiation condition holds, a suitable numerical method has to ensure that this condition is not violated. For example, FEMs need the application of special techniques to fulfill this condition that, on the contrary, is implicitly fulfilled by BEM; hence a suitable coupling of both these techniques, when applicable, gives undoubted advantages $[17,18]$.

In principle, both frequency-domain and time-domain BEM can be used for hyperbolic initial-boundary value problems [19-22]. Space-time BEM has the advantage that it directly yields the unknown time-dependent quantities. In this last approach, the construction of the BIEs, via representation formula in terms of single and double layer potentials, uses the fundamental solution of the hyperbolic partial differential equation and jump relations [23-25]. The mathematical background of time-dependent boundary integral equations is summarized by M. Costabel in [26].

For the numerical solution of the damped wave equation in 1D unbounded media, we have already considered in $[17,18,27]$ the extension of the so- 


\section{Energetic BEM ...}

called energetic BEM, introduced for the undamped wave equation in several space dimensions [28-30]. The analysis carried out for 1D damped wave propagation problems allowed to fully understand the approximation technique for what concerns marching on time, avoiding space integration with BEM singular kernels and it was considered as a touchstone for the extension to higher space dimensions, which is done here for the $2 \mathrm{D}$ case. Also in this context, the energetic approach gives consistent approximations and accurate numerical solutions.

Energetic BEM is based on a weak formulation directly expressed in the space-time domain, thus avoiding the use of the Laplace transform and of its inversion suggested in [23].

The paper is structured as follows: at first, we present the differential model problem on an unbounded 2D domain and its energetic boundary weak formulation, then we illustrate the consequent BEM discretization, highlighting problems arising in the numerical integration of singular space-time integrals. At last, a wide variety of significant numerical benchmarks are introduced and discussed, showing, from a numerical point of view, stability and accuracy of the obtained numerical solutions, in particular for simulations over large time intervals.

\section{Model problem and its weak boundary integral formulation}

Here we will consider a Dirichlet problem in a bounded time interval $[0, T]$ for the damped wave equation exterior to an obstacle, given by an open arc $\Gamma \subset \mathbb{R}^{2}$ :

$$
\begin{array}{lll}
\text { (1) } & {\left[\Delta u-\frac{1}{c^{2}} u_{t t}-\frac{2 D}{c^{2}} u_{t}-\frac{P}{c^{2}} u\right](\mathbf{x}, t)=0,} & \mathbf{x} \in \mathbb{R}^{2} \backslash \Gamma, t \in(0, T] \\
(2) & u(\mathbf{x}, 0)=u_{t}(\mathbf{x}, 0)=0, & \mathbf{x} \in \mathbb{R}^{2} \backslash \Gamma, \\
(3) & u(\mathbf{x}, t)=g(\mathbf{x}, t), & \mathbf{x} \in \Gamma, t \in(0, T],
\end{array}
$$

where $c$ is the propagation velocity of a perturbation inside the domain, $D$ and $P$ are, respectively, the viscous and material damping coefficients ${ }^{\mathrm{a}}$. Note that if $c$ is measured in $\mathrm{m} / \mathrm{s}, D$ is measured in $s^{-1}$ and $P$ in $s^{-2}$. Equation (1) appears in different contexts, from electric transmission to sound propagation, from primary or secondary seismic wave propagation in presence of intrinsic attenuation to quantum field theory [31-34].

\footnotetext{
${ }^{a}$ When mechanical systems vibrate in a fluid medium, the resistance offered by the fluid to the moving body causes energy to be dissipated. The amount of dissipated energy depends on many factors: in viscous damping, the damping force is proportional to the velocity of the vibrating body; on the contrary, material damping acts like a linear restoring force (such as due to a spring) that is proportional to the displacement.
} 


\section{A.Aimi et al.}

When $D=P=0$ the given PDE collapses to the classic wave equation. The boundary datum $g(\mathbf{x}, t)$ represents the value of the excitation field over $\Gamma$.

Since we want to discretize the above problem using BEM, we have to rewrite it in a boundary integral form. This can be done using classical arguments and the knowledge of the fundamental solution of the 2D damped wave operator. Hence, we start writing the single-layer representation of the solution of (1)-(3):

(4) $u(\mathbf{x}, t)=\int_{\Gamma} \int_{0}^{t} G(\mathbf{x}-\boldsymbol{\xi}, t-\tau) \phi(\boldsymbol{\xi}, \tau) d \tau d \gamma_{\boldsymbol{\xi}}, \quad \mathbf{x} \in \mathbb{R}^{2} \backslash \Gamma, t \in(0, T]$

where the unknown density $\phi=\left[\frac{\partial u}{\partial \mathbf{n}}\right]_{\Gamma}$ represents the time history of the jump of the normal derivative of $u$ along $\Gamma$ and

$$
G(\mathbf{x}, t)=\left\{\begin{array}{l}
\frac{c}{2 \pi} e^{-D t} \frac{\cos \left(\frac{\sqrt{P-D^{2}}}{c} \sqrt{c^{2} t^{2}-\|\mathbf{x}\|^{2}}\right)}{\sqrt{c^{2} t^{2}-\|\mathbf{x}\|^{2}}} H[c t-\|\mathbf{x}\|], P \geq D^{2} \\
\frac{c}{2 \pi} e^{-D t} \frac{\cosh \left(\frac{\sqrt{D^{2}-P}}{c} \sqrt{c^{2} t^{2}-\|\mathbf{x}\|^{2}}\right)}{\sqrt{c^{2} t^{2}-\|\mathbf{x}\|^{2}}} H[c t-\|\mathbf{x}\|], P \leq D^{2}
\end{array}\right.
$$

is the forward fundamental solution of the $2 \mathrm{D}$ damped wave operator, with $H[\cdot]$ the Heaviside distribution. Definition (5) switches from $\cos (\cdot)$ to $\cosh (\cdot)$ depending on the reciprocal magnitude of $P$ and $D^{2}$ : when $P>D^{2}$ we are in the so-called underdamping configuration, when $P<D^{2}$ we are in overdamping configuration, while the separation state $P=D^{2}$, referred to the vanishing of both $\cos (\cdot)$ and $\cosh (\cdot)$ arguments, is called critical damping. The usefulness of the two expressions in (5) depends on the problem at hand: to keep the vibrations as long as possible (as for instance in tuning fork), it is preferable to construct a physical system with $P>D^{2}$; on the contrary, to dampen oscillation as soon as possible or to avoid overshooting a target configuration, it is necessary to have $P<D^{2}$.

Note that in the limit for $D, P$ tending to $0, G(\mathbf{x}, t)$ tends to the fundamental solution of the $2 \mathrm{D}$ undamped wave operator, i.e.

$$
G_{0}(\mathbf{x}, t)=\frac{c}{2 \pi} \frac{H[c t-\|\mathbf{x}\|]}{\sqrt{c^{2} t^{2}-\|\mathbf{x}\|^{2}}} .
$$

Now, it is clear that if we want to recover the solution of the differential problem at any point outside the obstacle and at any time instant, we have to proceed with a post-processing phase provided that we know the density 


\section{Energetic BEM ...}

function $\phi(\mathbf{x}, t)$. Hence, since the extension of formula (4) for $\mathbf{x}$ tending to $\Gamma$ is continuous, the unknown density $\phi$ can be determined via the assigned Dirichlet boundary condition (3). This results in the space-time BIE

$$
\int_{\Gamma} \int_{0}^{t} G(\mathbf{x}-\boldsymbol{\xi}, t-\tau) \phi(\boldsymbol{\xi}, \tau) d \tau d \gamma_{\boldsymbol{\xi}}=g(\mathbf{x}, t), \quad \mathbf{x} \in \Gamma, t \in[0, T],
$$

which can be written with the compact notation

$$
V \phi=g .
$$

Existence and uniqueness of the solution of the Eq. (8) are proved in [35]. The energetic weak formulation of problem (8) is defined similarly as in [29] and it can be deduced observing that, multiplying the PDE (1) by $u_{t}$, integrating over $[0, T] \times\left(\mathbb{R}^{2} \backslash \Gamma\right)$ and using integration by parts in space, one obtains that the energy $\mathcal{E}(u, T)$ of the solution $u$ at the final time of analysis $T$, defined by

$\frac{1}{2} \int_{\mathbb{R}^{2} \backslash \Gamma}\left[\left\|\nabla_{\mathbf{x}} u(\mathbf{x}, T)\right\|^{2}+\frac{1}{c^{2}} u_{t}^{2}(\mathbf{x}, T)+\frac{P}{c^{2}} u^{2}(\mathbf{x}, T)+\frac{4 D}{c^{2}} \int_{0}^{T} u_{t}^{2}(\mathbf{x}, t) d t\right] d \gamma_{\mathbf{x}}$

can be rewritten as

$$
\mathcal{E}(u, T)=\int_{\Gamma} \int_{0}^{T} u_{t}(\mathbf{x}, t)\left[\frac{\partial u}{\partial \mathbf{n}}\right]_{\Gamma}(\mathbf{x}, t) d t d \gamma_{\mathbf{x}} .
$$

Hence, remembering the nature of our BIE which is expressing the time history of $u$ over $\Gamma$, we can derive it w.r.t. time and write down the energetic weak problem associated to (8) as:

find $\phi \in L^{2}(\Gamma \times[0, T])$ such that

$$
\int_{\Gamma} \int_{0}^{T}(V \phi)_{t}(\mathbf{x}, t) \psi(\mathbf{x}, t) d t d \gamma_{\mathbf{x}}=\int_{\Gamma} \int_{0}^{T} g_{t}(\mathbf{x}, t) \psi(\mathbf{x}, t) d t d \gamma_{\mathbf{x}},
$$

where $\psi$ is a suitable test function, belonging to the same functional space of $\phi$. With an integration in the sense of distributions, we can equivalently write

$$
\int_{\Gamma} \int_{0}^{T}(V \phi)(\mathbf{x}, t) \psi_{t}(\mathbf{x}, t) d t d \gamma_{\mathbf{x}}=\int_{\Gamma} \int_{0}^{T} g(\mathbf{x}, t) \psi_{t}(\mathbf{x}, t) d t d \gamma_{\mathbf{x}}
$$

Remark. The theoretical analysis of the quadratic form coming from the left-hand side of (11) was carried out for $P=D=0$ in [29] where, under suitable hypothesis, coercivity was proved with some technicalities. This 
A.Aimi et al.

allowed us to deduce stability and convergence of the related Galerkin approximate solution, which in this paper, for the case of non-trivial damping coefficients, will be verified from a numerical point of view. On the contrary, these properties are not guaranteed by the classical collocation method (see $[9,28])$.

\section{Energetic BEM discretization}

We consider on the obstacle $\Gamma$, a boundary mesh constituted by $M_{\Delta x}$ straight elements $\left\{e_{1}, \cdots, e_{\left.M_{\Delta x}\right\}}\right.$, with length $\left(e_{i}\right) \leq \Delta x, e_{i} \cap e_{j}=\emptyset$ if $i \neq j$ and such that $\bigcup_{i=1}^{M_{\Delta x}} \bar{e}_{i}$ coincides with $\bar{\Gamma}$ if the obstacle is (piece-wise) linear, or is a suitable approximation of $\bar{\Gamma}$, otherwise. The functional background compels one to choose space shape functions belonging to $L^{2}(\Gamma)$, although higher degree shape functions can be used. Hence we use standard piece-wise constant polynomial boundary element functions $w_{j}(\mathbf{x}), j=1, \cdots, M_{\Delta x}$, suitably defined in relation to the introduced mesh over $\Gamma$.

For time discretization we consider a uniform decomposition of the time interval $[0, T]$ with time step $\Delta t=T / N_{\Delta t}, N_{\Delta t} \in \mathbb{N}^{+}$, generated by the $N_{\Delta t}+1$ instants

$$
t_{k}=k \Delta t, \quad k=0, \cdots, N_{\Delta t},
$$

and we choose piece-wise constant time shape functions. Note that, for this particular choice, our shape functions, denoted with $v_{k}(t), k=0, \cdots, N_{\Delta t}-$ 1 , will be defined as

$$
v_{k}(t)=H\left[t-t_{k}\right]-H\left[t-t_{k+1}\right]
$$

Hence, the approximate solution of the problem at hand will be expressed as

$$
\phi(\mathbf{x}, t) \simeq \sum_{k=0}^{N_{\Delta t}-1} \sum_{j=1}^{M_{\Delta x}} \alpha_{j}^{(k)} w_{j}(\mathbf{x}) v_{k}(t)
$$

The Galerkin BEM discretization coming from energetic weak formulation (12) produces the linear system

$$
A \alpha=b,
$$

of order $M_{\Delta x} \cdot N_{\Delta t}$, where matrix $A$ has a block lower triangular Toeplitz structure. Each block has dimension $M_{\Delta x}$. If we indicate with $A^{(\ell)}$ the 


\section{Energetic BEM ...}

block obtained when $t_{h}-t_{k}=\ell \Delta t, \ell=0, \ldots, N_{\Delta t}-1$, the linear system can be written as

$$
\left(\begin{array}{ccccc}
A^{(0)} & 0 & 0 & \cdots & 0 \\
A^{(1)} & A^{(0)} & 0 & \cdots & 0 \\
A^{(2)} & A^{(1)} & A^{(0)} & \cdots & 0 \\
\cdots & \cdots & \cdots & \cdots & 0 \\
A^{\left(N_{\Delta t}-1\right)} & A^{\left(N_{\Delta t}-2\right)} & \cdots & A^{(1)} & A^{(0)}
\end{array}\right)\left(\begin{array}{c}
\alpha^{(0)} \\
\alpha^{(1)} \\
\alpha^{(2)} \\
\vdots \\
\alpha^{\left(N_{\Delta t}-1\right)}
\end{array}\right)=\left(\begin{array}{c}
b^{(0)} \\
b^{(1)} \\
b^{(2)} \\
\vdots \\
b^{\left(N_{\Delta t}-1\right)}
\end{array}\right)
$$

where

$$
\alpha^{(\ell)}=\left(\alpha_{j}^{(\ell)}\right) \quad \text { and } \quad b^{(\ell)}=\left(b_{j}^{(\ell)}\right), \quad j=1, \ldots, M_{\Delta x} .
$$

The solution of (17) is obtained with a block forward substitution, i.e. at every time instant $t_{\ell}=\ell \Delta t, \ell=0, \cdots, N_{\Delta t}-1$, we solve a reduced linear system of the type

$$
A^{(0)} \alpha^{(\ell)}=b^{(\ell)}-\left(A^{(1)} \alpha^{(\ell-1)}+\cdots+A^{(\ell)} \alpha^{(0)}\right) .
$$

Procedure (19) is a time-marching technique, where the only matrix to be inverted is the symmetric positive definite diagonal block $A^{(0)}$, while all the other blocks are used to update at every time step the right-hand side. Owing to this procedure we can construct and store only the blocks $A^{(0)}, \cdots, A^{\left(N_{\Delta t}-1\right)}$ with a considerable reduction of computational cost and memory requirement [29].

\section{Multiple integration issues}

In (16), matrix elements are integrals of the form

$$
\int_{\Gamma} \int_{0}^{T} w_{i}(\mathbf{x})\left(v_{h}\right)_{t}(t) \int_{\Gamma} \int_{0}^{t} G(\mathbf{x}-\boldsymbol{\xi}, t-\tau) w_{j}(\boldsymbol{\xi}) v_{k}(\tau) d \tau d \gamma_{\boldsymbol{\xi}} d t d \gamma_{\mathbf{x}}
$$

Specifying the choice made for time basis function, quadruple integral (20) results in a combination of triple integrals of the form

$$
\int_{\Gamma} w_{i}(\mathbf{x}) \int_{\Gamma} w_{j}(\boldsymbol{\xi}) \int_{0}^{t_{h}} G\left(\mathbf{x}-\boldsymbol{\xi}, t_{h}-\tau\right) H\left[\tau-t_{k}\right] d \tau d \gamma_{\boldsymbol{\xi}} d \gamma_{\mathbf{x}},
$$

or equivalently

$$
H\left[t_{h}-t_{k}\right] \int_{\Gamma} w_{i}(\mathbf{x}) \int_{\Gamma} w_{j}(\boldsymbol{\xi}) \int_{t_{k}}^{t_{h}} G\left(\mathbf{x}-\boldsymbol{\xi}, t_{h}-\tau\right) d \tau d \gamma_{\boldsymbol{\xi}} d \gamma_{\mathbf{x}} .
$$




\section{A.Aimi et al.}

The analysis of kernel weak singularities has been performed in detail for the case of the undamped wave equation, i.e. for $P=D=0$, in [29]; in particular the presence of the Heaviside distribution $H[c(t-\tau)-\|\mathbf{x}-\boldsymbol{\xi}\|]$, which represents the wavefront, and of the square root $\sqrt{c^{2}(t-\tau)^{2}-\|\mathbf{x}-\boldsymbol{\xi}\|^{2}}$ can cause a lot of numerical troubles that in [29] have been solved by suitable splitting of the outer integral over $\Gamma$ and using quadrature schemes which regularize integrand functions with mild singularities for the second nested integral in space variable [36]. These singularities appear after the inner time integration.

Since we expect a similar behavior for the damped kernel, we consider the expansion of $G(\mathbf{x}-\boldsymbol{\xi}, t-\tau)$ with respect to damping parameters, centered in $P=D=0$, and we rewrite $(22)$ as

$$
\begin{aligned}
& H\left[t_{h}-t_{k}\right] \int_{\Gamma} w_{i}(\mathbf{x}) \int_{\Gamma} w_{j}(\boldsymbol{\xi}) \int_{t_{k}}^{t_{h}}\left[G\left(\mathbf{x}-\boldsymbol{\xi}, t_{h}-\tau\right)\right. \\
& \left.-G_{0}\left(\mathbf{x}-\boldsymbol{\xi}, t_{h}-\tau\right)\right] d \tau d \gamma_{\boldsymbol{\xi}} d \gamma_{\mathbf{x}} \\
& +H\left[t_{h}-t_{k}\right] \int_{\Gamma} w_{i}(\mathbf{x}) \int_{\Gamma} w_{j}(\boldsymbol{\xi}) \int_{t_{k}}^{t_{h}} G_{0}\left(\mathbf{x}-\boldsymbol{\xi}, t_{h}-\tau\right) d \tau d \gamma_{\boldsymbol{\xi}} d \gamma_{\mathbf{x}},
\end{aligned}
$$

where the undamped kernel $G_{0}$ is defined in (6), in such a way that the problematic issues described above are confined in the second term, whose time integral can be evaluated analytically. The time integral of the first term is instead performed numerically, using a modified Gaussian rule [36]. At last, the outer integrals over $\Gamma$ are numerically treated by suitable quadrature schemes as in [29], to which the interested reader is referred. In particular, we use 32 quadrature nodes for the space cell and from 32 to 128 quadrature nodes for the time cell, depending on the accuracy we need to catch the highly oscillatory behavior of the approximate solutions, as it will be clear in the next Section.

Remark. At present, nothing have been done to optimize the computational costs, but the elapsed time for the longest simulation is not greater than one hour on a standard personal computer. In this sense, a remarkable computational saving could be achieved parallelizing the generation of matrix blocks $A^{(\ell)}, \ell=0, \cdots, N_{\Delta t}-1$ : they are independent of each other and therefore they could be computed simultaneously.

Further, the computational cost of the post-processing phase and of the linear system (17) generation and solution are not strictly related. In fact, while post-processing involves only double integrals with non singular kernel as expressed in (4) and it linearly depends on the number of space-time points at which we want to evaluate the solution of the original differential problem, the linear system computation is made heavy mainly by the 


\section{Energetic BEM ...}

evaluation of triple integrals (22) with their weak singularities.

\section{Numerical results}

In the following, we will present and discuss several numerical results obtained, for 2D Dirichlet exterior problems, by energetic Galerkin BEM. As already said in Section 2, problem (1)-(3) can model damping phenomena in very different physics and engineering contexts. As a consequence, velocity $c$ and damping parameters $P$ and $D$, often tuned with respect to the value of $c$, may assume very different values in a range between 0 and some thousands. But changing the value of the velocity results in rescaling time-space domain and related discretization parameters, as done for the energetic BEM in a previous paper [28]. Hence, here, we chose to show the reliability of the proposed numerical method varying the damping coefficients in a suitably wide range, having fixed $c=1$.

In the first benchmark, we consider the problem (1)-(3) with $\Gamma=$ $\{\mathbf{x}=(x, 0) \mid x \in[-0.5,0.5]\}$ and the Dirichlet boundary datum

$$
g(x, t)=-H[t] f(t) x, \quad \text { where } \quad f(t)= \begin{cases}\sin ^{2}(4 \pi t), & \text { if } \quad 0 \leq t \leq \frac{1}{8} \\ 1, & \text { if } \quad t \geq \frac{1}{8}\end{cases}
$$

taken from [29].

At first, we choose a uniform decomposition of $\Gamma$ in 40 straight elements $(\Delta x=0.025)$ and we set $\Delta t=0.025$. In Figure 1 , we show the time history of the solutions $\phi(x, t)$ on the straight element $e_{10}$ and on the time interval $[0,2]$, for $P=0$ varying $D=0,0.5,1,2,5,10,20$ on the top and for $D=0$ varying $P=0,0.1,1,10,20$ at bottom. Note the effects of increasing viscous and material damping, which substantially change the aspect of the solution related to the classical wave equation, visible in the graphs for trivial parameters.

During the turbulent phase, the BIE solutions have a peak in the time interval $[0,1 / 8]$ in accordance with the time evolution of Dirichlet datum. Then the datum becomes constant, forcing the solution to be trivial until it receives solicitation by an endpoint of the boundary that behaves like a wave source: on $e_{10}$, the left-end side of the boundary influences the BIE solution at $t=0.25$ and then, at $t=0.75$ the wave coming from the right-end side arrives... Of course, the higher the viscous damping parameter D in Figure 1 (top), the more the reflected waves from the endpoints are damped, and the above described phenomenon is negligible. On the contrary, the higher the material damping parameter $P$ in Figure 1 (bottom), the more the reflected waves from the endpoints are amplified, as in a bigger oscillation. 


\section{A.Aimi et al.}

Then, in order to observe longtime behavior of the solution and to numeri-
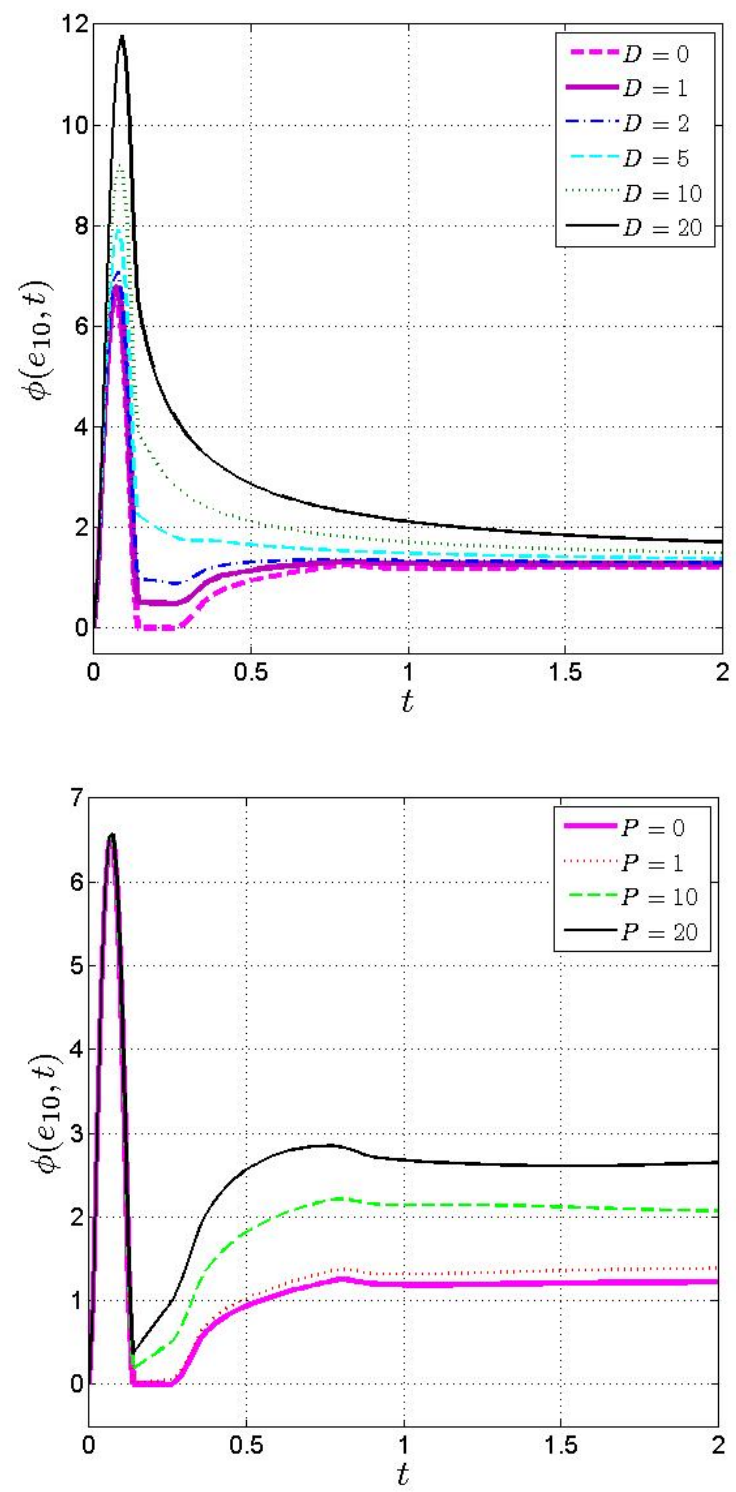

Figure 1. Time history of $\phi(x, t)$ on element $e_{10}$, for $P=0$ (top) and $D=0$ (bottom). cally check longtime stability of the energetic formulation (11), we choose a uniform decomposition of $\Gamma$ in 10 subintervals $(\Delta x=0.1)$ and enlarge the observation time interval, fixing $T=60$ and $\Delta t=0.1$. Since the Dirichlet datum becomes independent of time, for $P=0$ and $D \geq 0$ we expect that the BIE transient solution $\phi(x, t)$ on $\Gamma$ tends to the stationary one $\phi_{\infty}(x)$ 


\section{Energetic BEM ...}

(Fig. 2), i.e. the solution of the BIE related to the following Dirichlet problem for the Laplace equation:

$$
\begin{array}{ll}
\Delta u_{\infty}(x)=0, & x \in \mathbb{R}^{2} \backslash \Gamma \\
u_{\infty}(x)=-x, & x \in \Gamma \\
u_{\infty}(x)=O(1), & \|x\| \rightarrow \infty
\end{array}
$$

Looking at the graphs of the time history of $\left\|\phi(\cdot, t)-\phi_{\infty}(\cdot)\right\|_{L^{1}(\Gamma)}$, shown

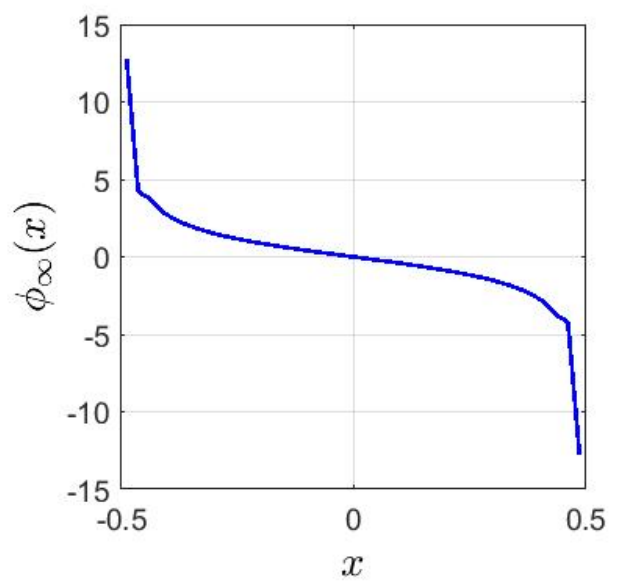

Figure 2. BIE stationary solution $\phi_{\infty}(x)$ on $\Gamma$

in Figure 3, we observe the expected convergence. In particular, curves for $D>0$ have the same slope, while the fastest convergence is related to the case of undamped wave equation, i.e. for $D=0$. Note that peaks appearing in Figure 3 are due to an intersection between transient and stationary solutions.

The convergence of the transient solution to the stationary one can be observed also outside the obstacle: once the unknown density over $\Gamma$ is obtained using energetic BEM, in the post-processing phase the representation formula (4) allows to evaluate the approximate solution around the obstacle. In Figures 4-6 we show snapshots of the transient solution for $P=D=0$ around $\Gamma$, evaluated at $t=2,4,6,8,10$, and the corresponding stationary solution. After the wavefront has left the considered area, the transient approximate solution is going to overlap the stationary one. In Figure 7 , time history of the difference in $L^{2}$ norm between transient and stationary solutions around the obstacle is presented, for $P=D=0$. 
A.Aimi et al.

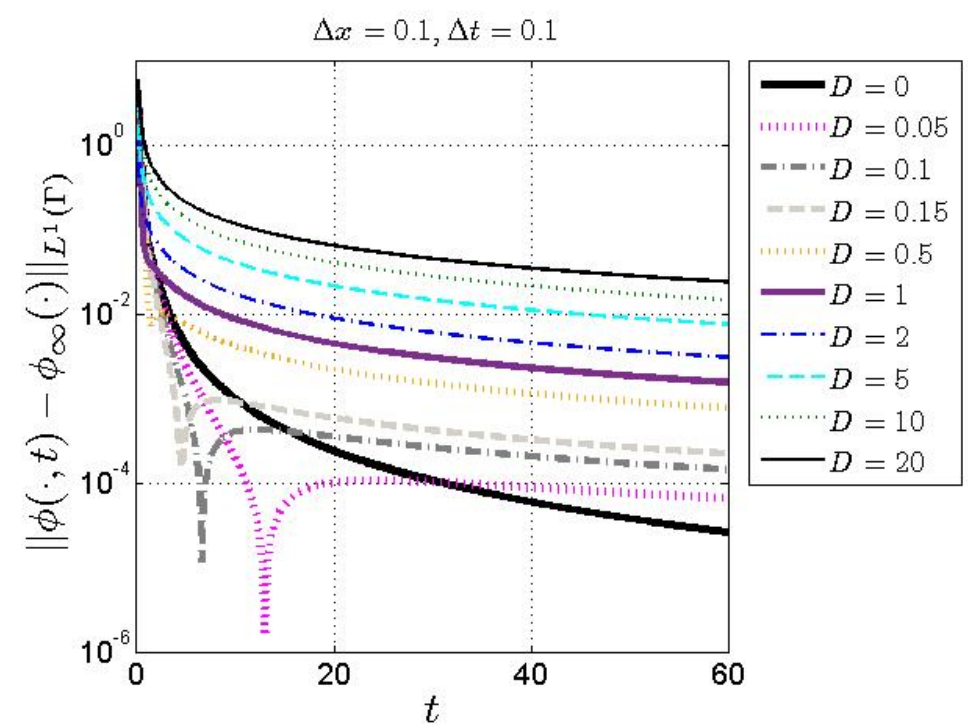

Figure 3. Convergence of damped BIE solution, for $P=0$ and $D \geq 0$
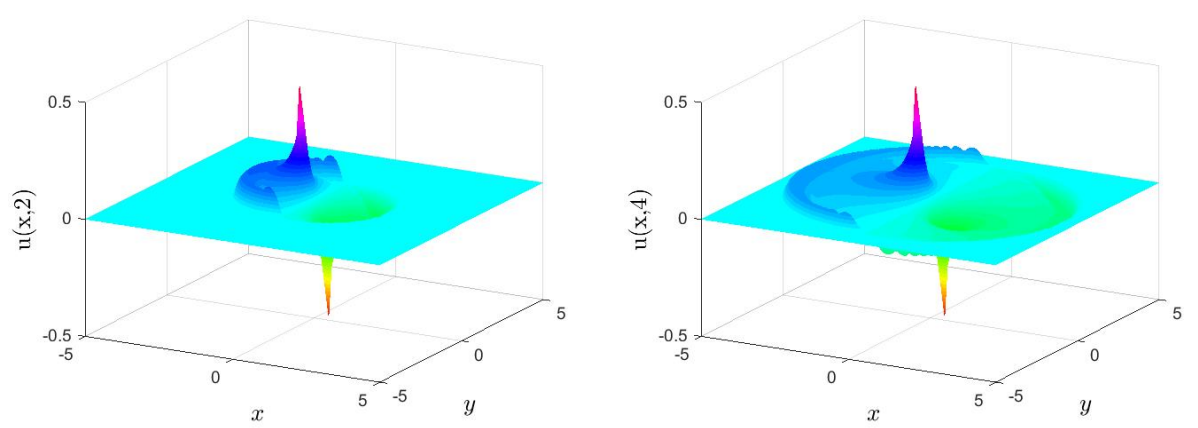

Figure 4. Snapshots of the transient solution $u(x, t)$ for $P=D=0$ around $\Gamma$, evaluated at $t=2$ and $t=4$.

The same analysis for the post-processing phase has been done in the case of $P=0, D=1$ : results are presented in Figures 8-10 and in Figure 11. Note that for active viscous damping coefficient, the wavefront leaving the obstacle is almost negligible and the convergence to the stationary solution 


\section{Energetic BEM ...}
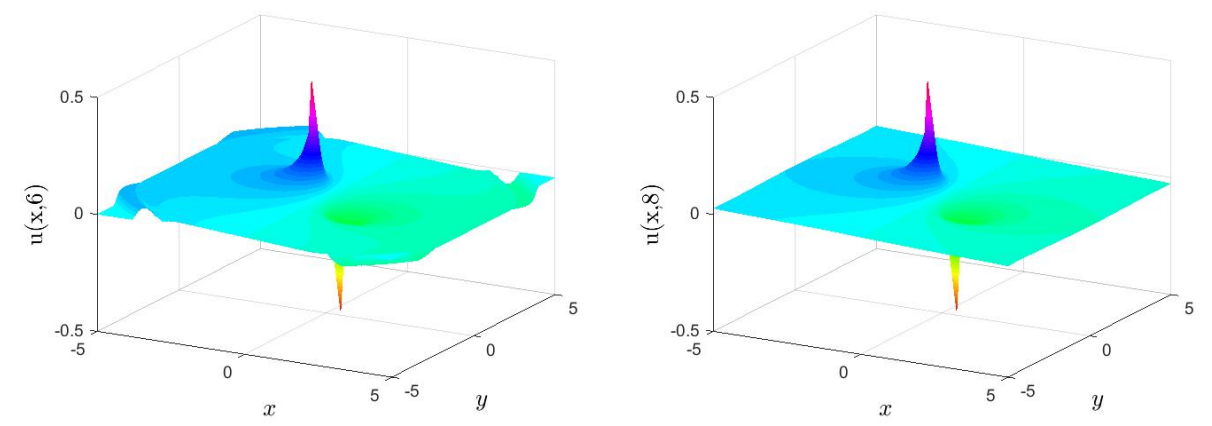

Figure 5. Snapshots of the transient solution for $P=D=0$ around $\Gamma$, evaluated at $t=6,8$.
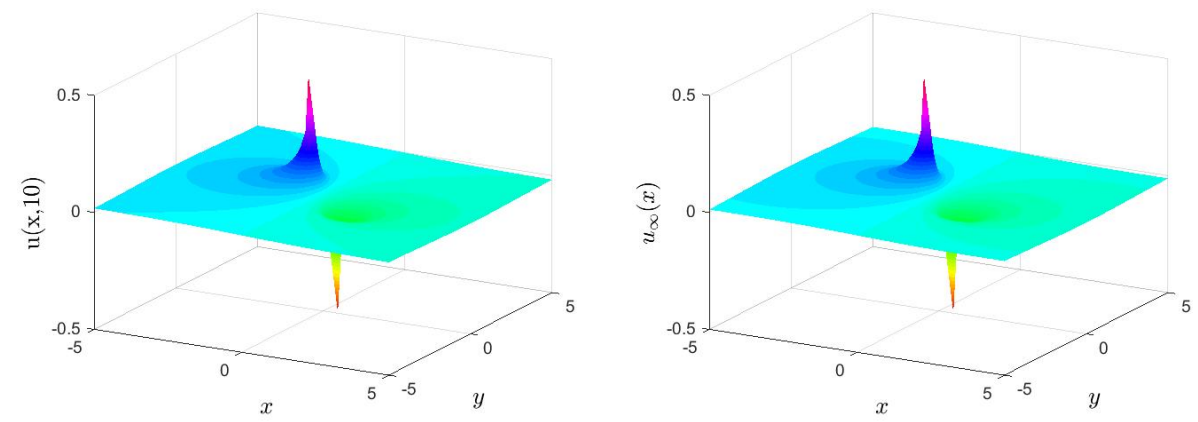

Figure 6. Transient solution for $P=D=0$ around $\Gamma$ evaluated at $t=10$ and stationary one.

is slower than in the previous case, related to trivial damping coefficients.

Analogously, for $D=0$ and $P \geq 0$, we expect that the transient solution $\phi(x, t)$ on $\Gamma$ tends to the stationary one $\phi_{\infty, k}(x)$ (Fig. 12), i.e. the solutions of the BIE related to the following Dirichlet problem for the Helmholtz equation:

$$
\begin{array}{ll}
\Delta u_{\infty, k}(x)+k^{2} u_{\infty, k}(x)=0, & x \in \mathbb{R}^{2} \backslash \Gamma \\
u_{\infty, k}(x)=-x, & x \in \Gamma \\
u_{\infty, k}(x)=O\left(\|x\|^{-1}\right), & \|x\| \rightarrow \infty
\end{array}
$$

with wave number $k=\sqrt{-P} / c^{2}$.

Looking at the graphs of the time history of $\left\|\phi(\cdot, t)-\phi_{\infty, k}(\cdot)\right\|_{L^{1}(\Gamma)}$, for 
A.Aimi et al.

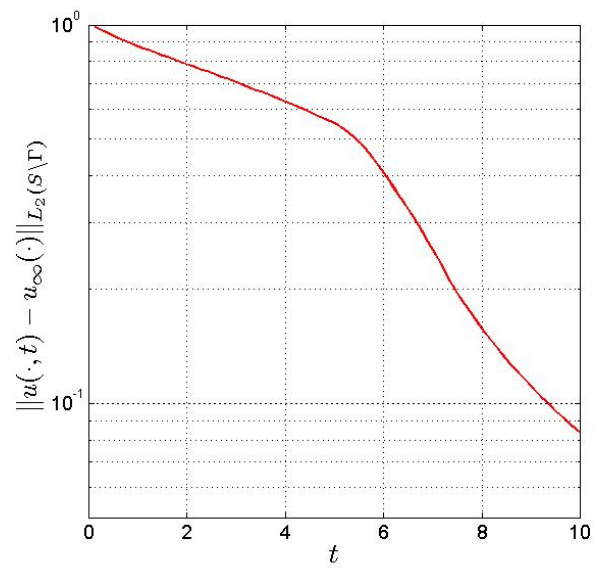

Figure 7. Time history of the difference in $L^{2}$ norm between transient and stationary solution around $\Gamma$, for $P=D=0$.
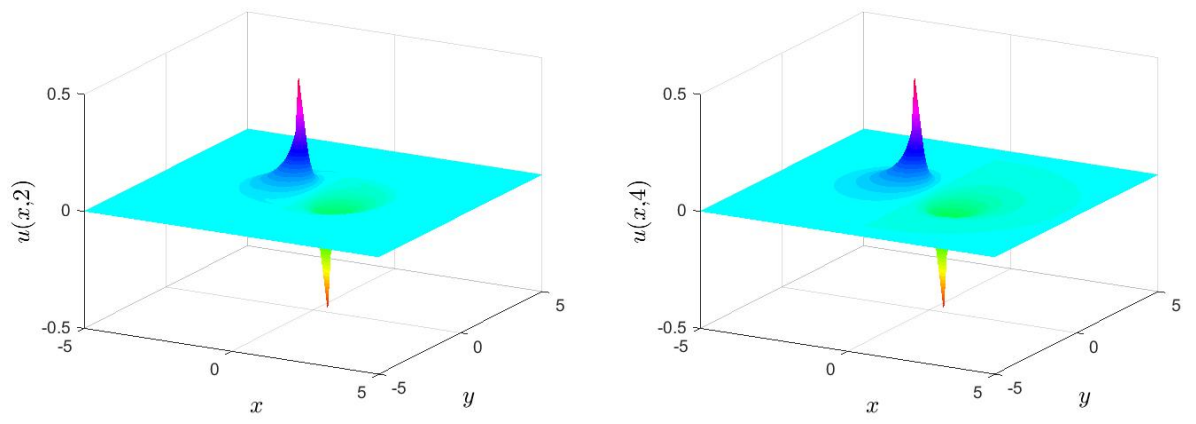

Figure 8. Snapshots of the transient solution for $P=0, D=1$ around $\Gamma$ evaluated, in $t=2,4$.

$D=0$ and $P=0.1,1,10,20$, in Figure 13, we observe the expected convergence, that becomes more oscillating for increasing values of parameter $P$, even if all the curves present the same global decay.

Also for both positive viscous and material damping coefficients, we expect convergence to the related Helmholtz stationary BIE problem solution. In Figure 14 we show the case $D=0.1$ and $P=0.1,1,10,20$, while in Figure 15 we show the comparison of the convergence history for $D=0$ and $D=0.1$, fixing different values of $P$ : the slower convergence appears 


\section{Energetic BEM ...}
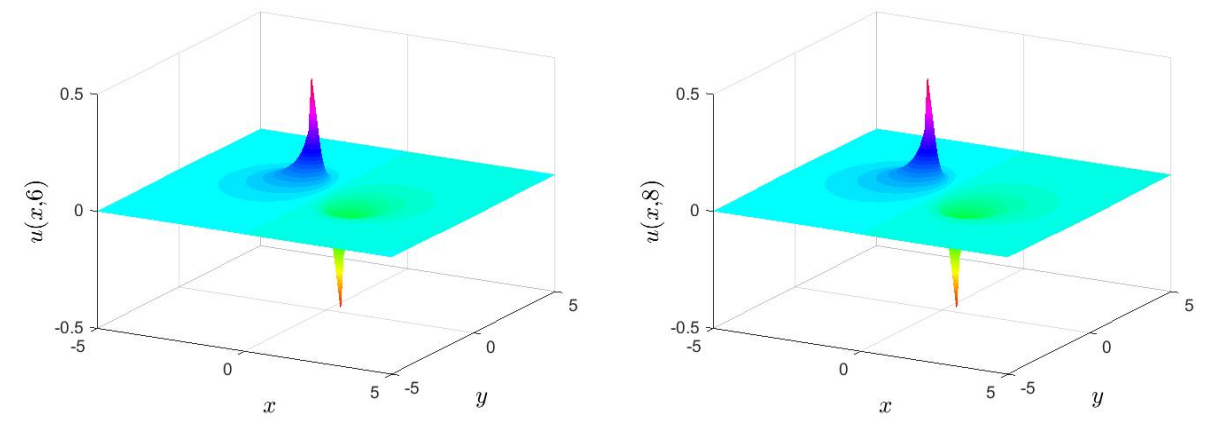

Figure 9. Snapshots of the transient solution for $P=0, D=1$ around $\Gamma$, evaluated in $t=6,8$.
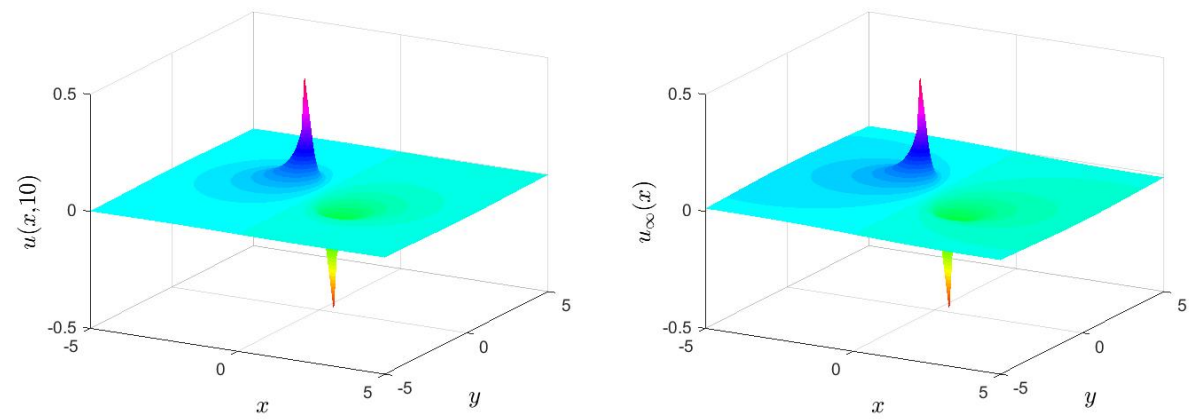

Figure 10. Transient solution for $P=0, D=1$ around $\Gamma$ evaluated in $t=10$ and stationary one.

for $D=0$. Note that simulations related to Figure 15 are all performed in underdamping configuration, while those in Figure 3 in overdamping configuration.

In general, as well known, if $P=D^{2}$ (critical damping) the physical model reaches the steady state as quickly as possible without oscillating; otherwise, in strictly connection with the two expressions defining the fundamental solution written in (5), if $P>D^{2}$ (underdamping) the decay combines with oscillations; if $P<D^{2}$ (overdamping) there is a decay to steady state without oscillations.

This behavior can be better explained looking at Figure 16: the convergence histories to the unique Helmholtz BIE solution for $k=\sqrt{-P}=i 0.1$ are plotted for different values of viscous damping $D$. When $D=0$ and $D=0.05$ we are in underdamping configuration and the higher $D$, the less 
A.Aimi et al.

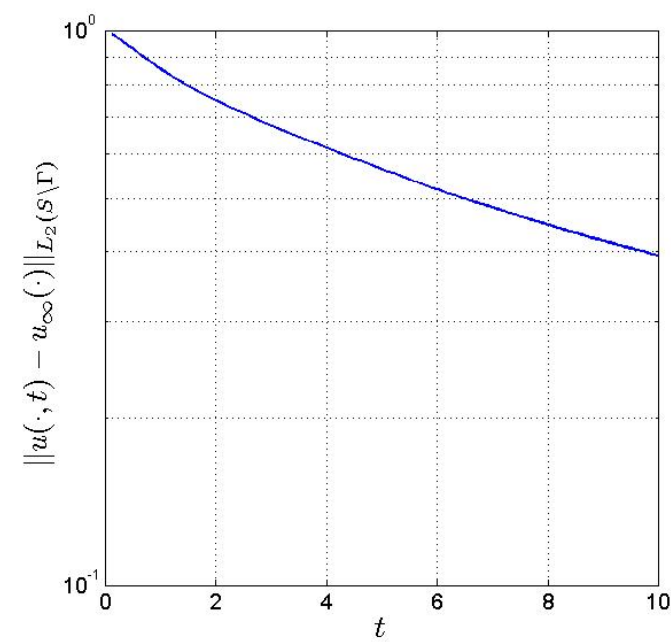

Figure 11. Time history of the difference in $L^{2}$ norm between transient and stationary solution around $\Gamma$, for $P=0, D=1$.

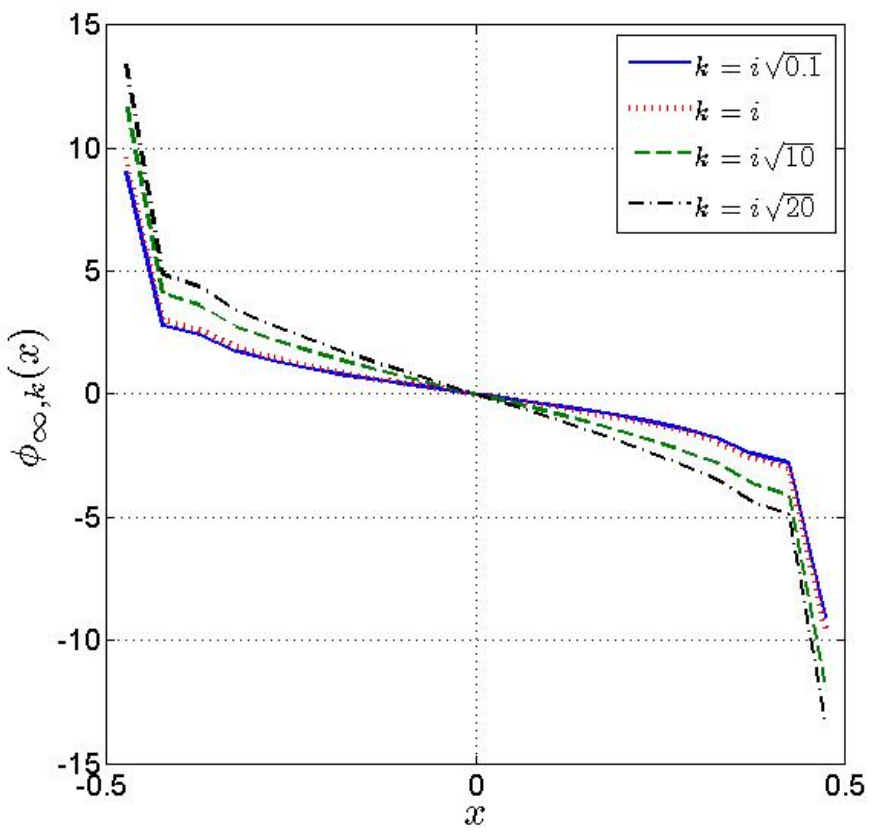

Figure 12. Stationary solutions $\phi_{\infty, k}(x)$ on $\Gamma$, for different values of $k$. 


\section{Energetic BEM ...}
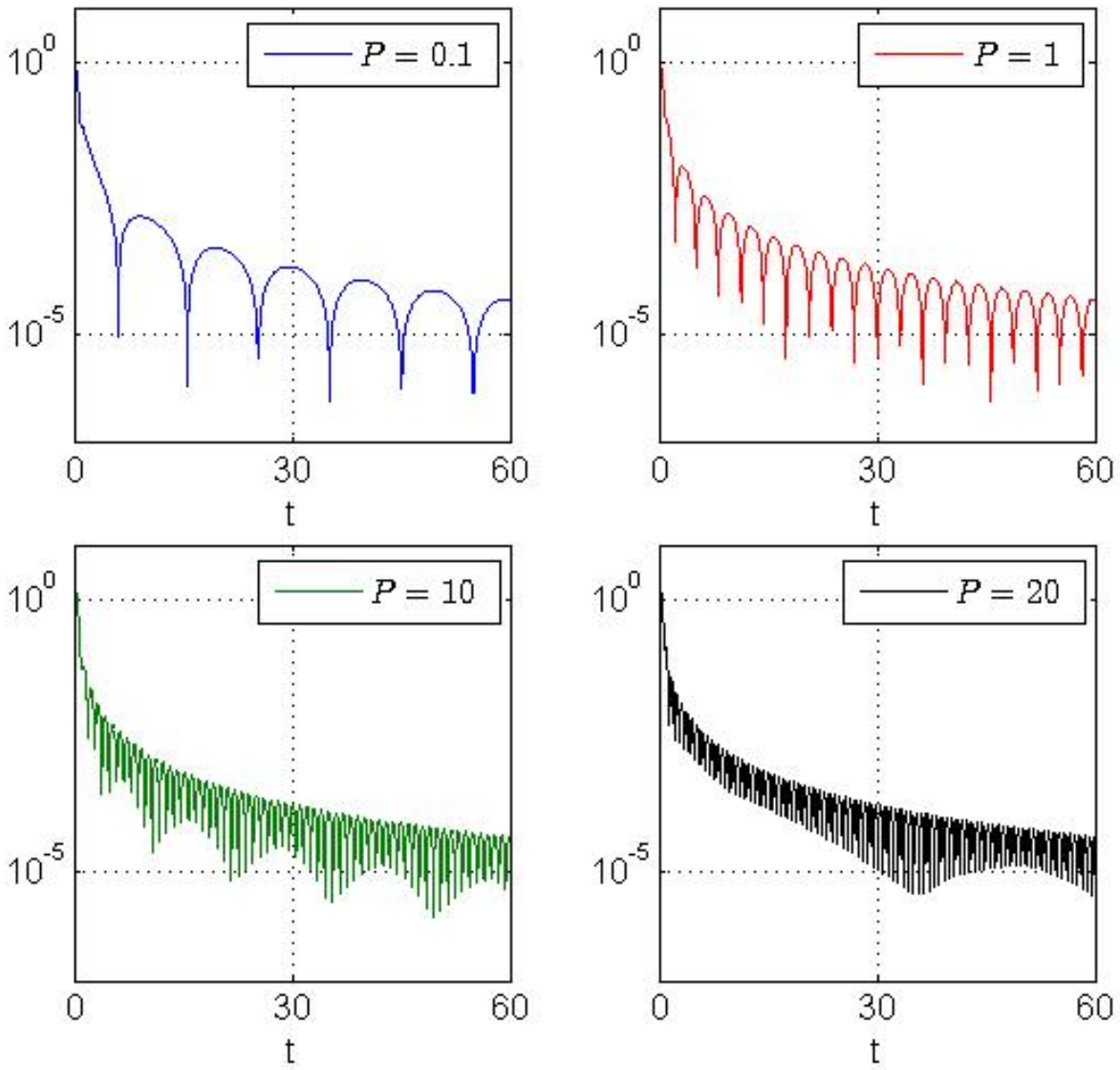

Figure 13. Convergence of damped BIE solution towards the related Helmholtz problem stationary BIE solution, when $D=0$ and $P=0.1,1,10,20$.

are the oscillations and the faster is the convergence to the steady state solution (continuous coloured lines). For $D=0.1$, when damping is critical, we observe the fastest convergence without oscillation (black line). Then, for growing $D$, we turn to the overdamping configuration and the higher the values of $D$, the slower is the convergence to the equilibrium without oscillations (dashed lines).

Further, in Figure 17, we give the same type of results for fixed $D=0.1$, 
A.Aimi et al.
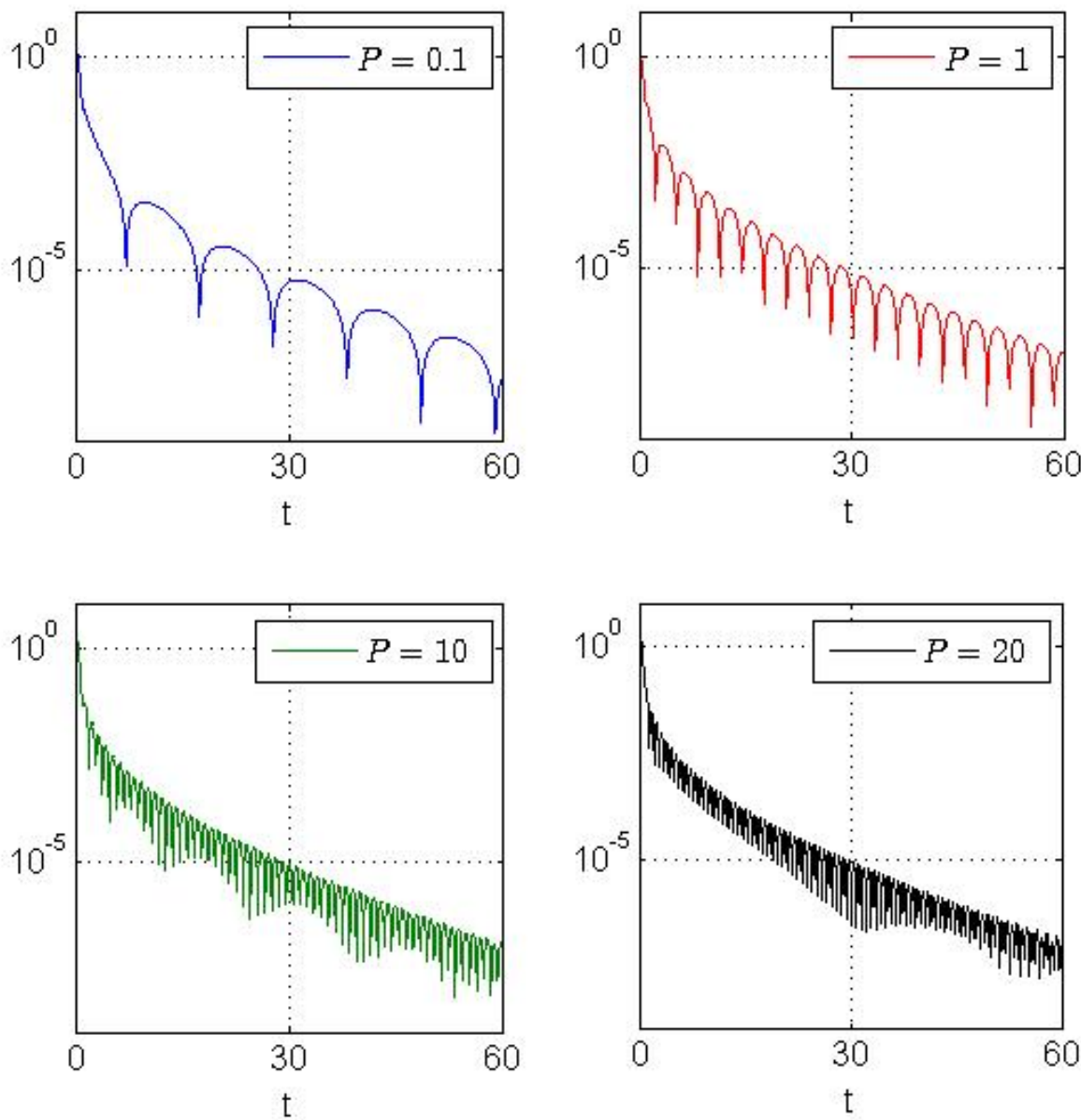

Figure 14. Convergence of damped BIE solution towards the related Helmholtz problem stationary BIE solution, when $D=0.1$ and $P=0.1,1,10,20$.

varying $P$, but here each transient solution converges to its own equilibrium. For growing $P<D^{2}$ (overdamping), the higher the material damping coefficient, the faster the convergence without oscillations (dashed lines). When critical damping is reached for $P=0.01$, we observe the fastest convergence without oscillation (black line). Then, for growing $P>D^{2}$ (underdamping), the decay combines with oscillations and the larger values of material damping $P$ the higher the frequency of oscillations (continuous coloured lines).

Finally, Figure 18 shows that, when $P=D^{2}$ (critical damping), the greater 


\section{Energetic BEM ...}
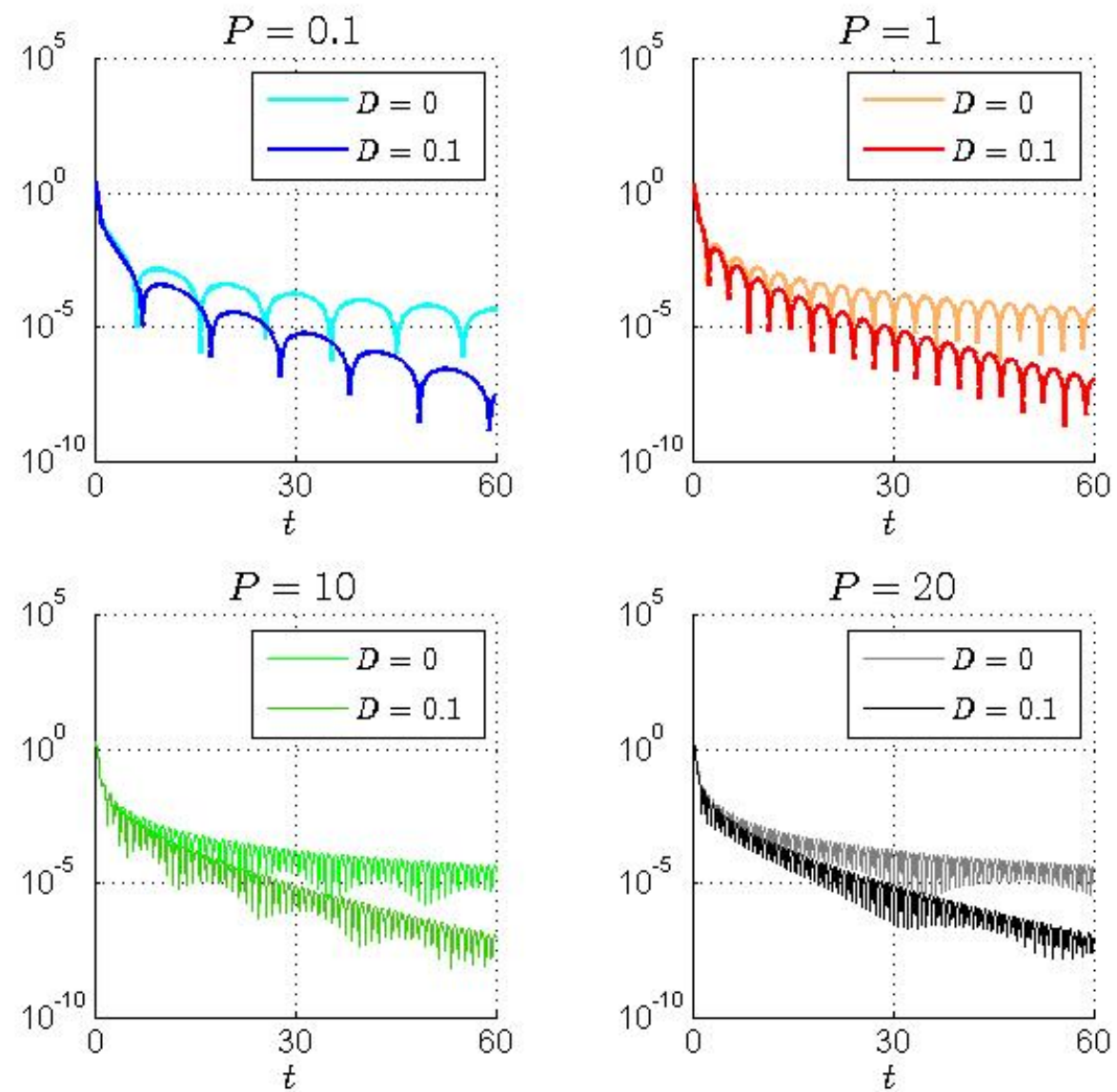

Figure 15. Comparison between convergence histories for $D=0$ and $D=0.1$, fixing different values of $P$.

the dissipation, the faster the convergence towards the related equilibrium. In that Figure the numerical accuracy threshold at $10^{-9}$ can be noticed, too.

In the second benchmark, we consider the problem (1)-(3) with $\Gamma=$ $\left\{\mathbf{x} \in \mathbb{R}^{2} \mid \mathbf{x}=(\cos \beta, \sin \beta), \beta \in[0, \pi]\right\}$ and the Dirichlet boundary datum, taken from [29],

$$
g(\beta, t)=H[t] f(t) \cos (\beta),
$$

with $f(\cdot)$ given in $(24)$. The velocity is still fixed as $c=1$. We choose a 
A.Aimi et al.

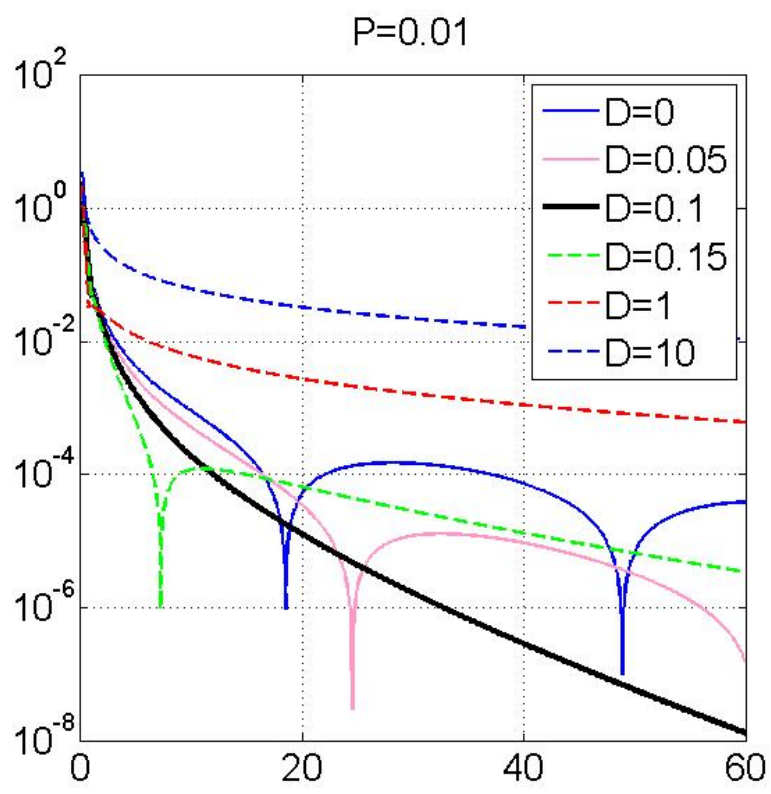

Figure 16. Comparison between convergence histories for fixed $P=0.01$, varying $D$.

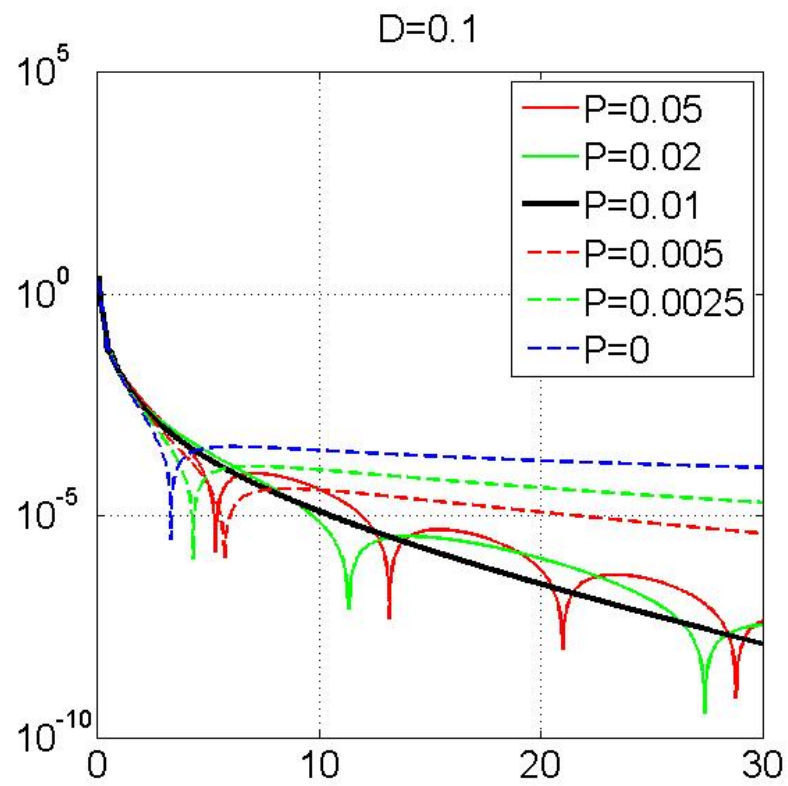

Figure 17. Comparison between convergence histories for fixed $D=0.1$, varying $P$. 


\section{Energetic BEM ...}

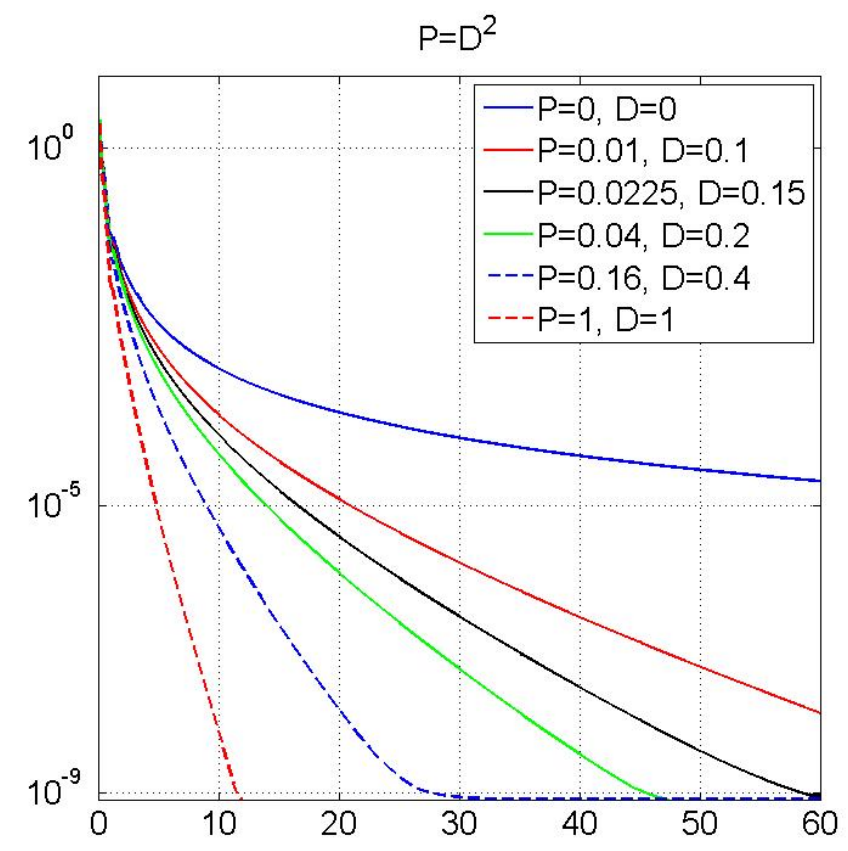

Figure 18. Comparison between convergence histories for different values of $P=D^{2}$.

uniform decomposition of $\Gamma$ in 40 straight elements $(\Delta x=\pi / 40)$ and we set $\Delta t=0.1$. The analysis is carried out on the time interval $[0,10]$. In Figure 19, we show the time history of the solutions $\phi(x, t)$ on the straight element $e_{10}$ for different values of damping parameters $P$ and $D$, while in Figure 20 the approximate solutions on $\Gamma$ at the final time instant of analysis are plotted. Note that for $P=0, D=0,1$ the transient BIE solutions tend to the stationary solution of the BIE related to the corresponding Laplace problem, while for $P=1, D=0,1$ the transient BIE solutions tend to the stationary solution of the BIE related to the corresponding Helmholtz problem, as in the first benchmark involving a straight obstacle $\Gamma$.

\section{Conclusions}

In this paper we have considered the so-called energetic BEM for the numerical solution of $2 \mathrm{D}$ damped wave propagation exterior problems equipped with Dirichlet boundary condition. The method was already considered for the numerical solution of the undamped wave equation in sev- 
A.Aimi et al.

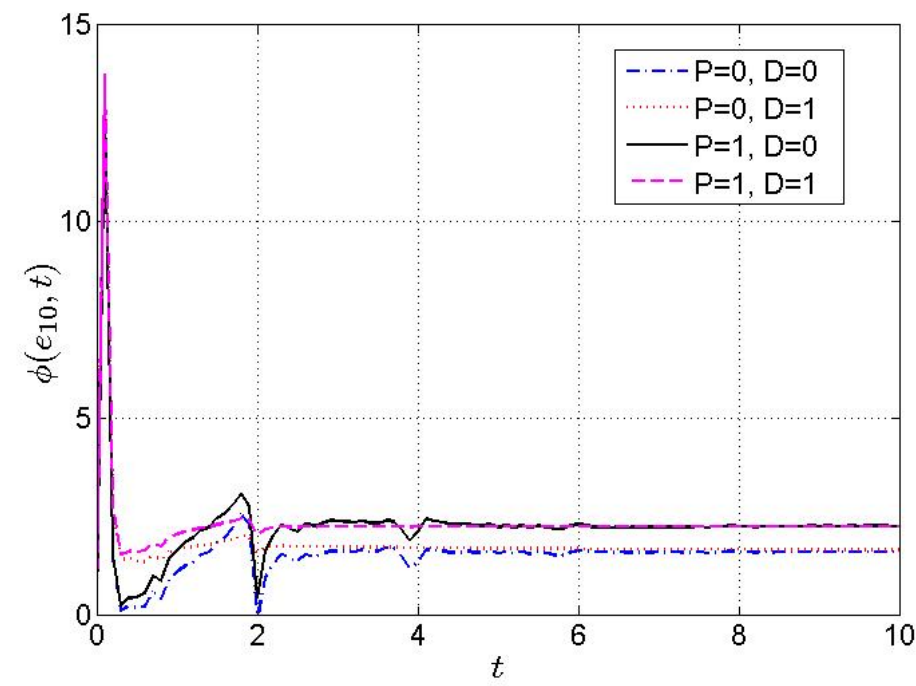

Figure 19. Time history of the solutions $\phi(x, t)$ on the straight element $e_{10}$, for different values of damping parameters $P$ and $D$.

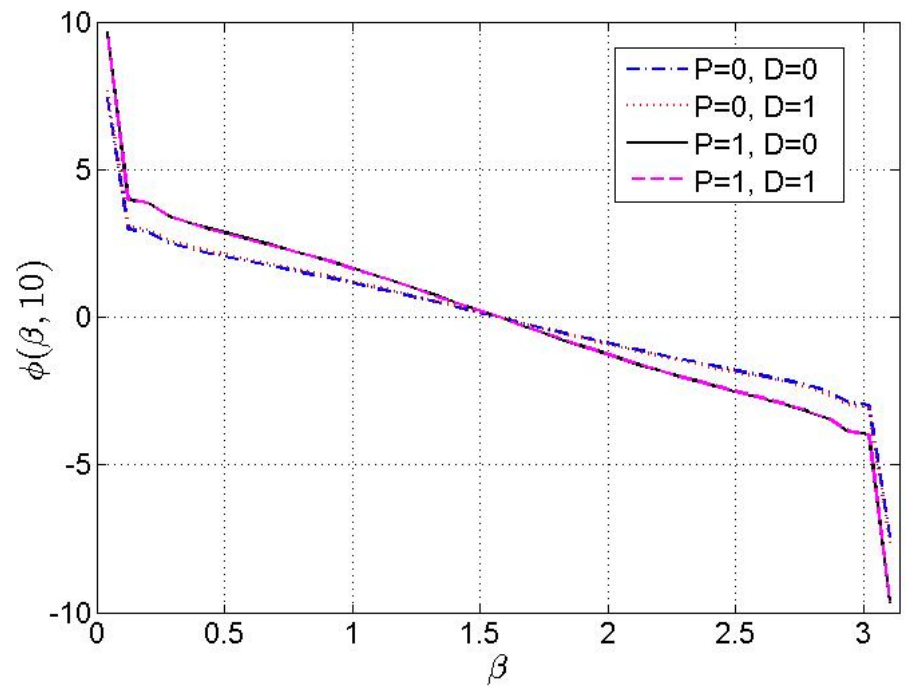

Figure 20. Approximate solutions on $\Gamma$ at the final time instant of analysis $T=10$, for different values of damping parameters $P$ and $D$.

eral space dimensions, revealing its accuracy and stability, also coupled with FEM. The presented numerical results confirm that these properties 


\section{Energetic BEM ...}

are maintained in presence of dissipation terms in the model problem as already highlighted in 1D simulations [17,27]. Ongoing research is focused on the analysis of strong and hyper-singular kernels obtained by normal derivation of the fundamental solution of the $2 \mathrm{D}$ damped wave operator and on the treatment of Neumann boundary conditions.

\section{REFERENCES}

1. M. P.M. and I. K.U., Theoretical Acoustic. McGraw-Hill, 1968.

2. D. Colton and R. Kress, Inverse Acoustic and Electromagnetic Scattering Theory. Springer, 2012.

3. L. Tsang, J. A. Kong, K.-H. Ding, and C. O. Ao, Scattering of Electromagnetic waves, Numerical Simulations. John Wiley \& Sons., 2004.

4. P. Banerjee and P. Butterfield, Boundary Element Methods in Engineering. McGraw-Hill U.K. Ltd., 1981.

5. F. Hartmann, Introduction to Boundary Element Theory Method in Engineering. McGraw-Hill, U.K. Ltd., 1981.

6. A. Aimi, M. Diligenti, C. Guardasoni, and S. Panizzi, Energetic BEMFEM coupling for wave propagation in layered media, Commun. Appl. Ind. Math., vol. DOI: 10.1685/journal.caim.438, 2013.

7. A. Aimi, L. Desiderio, M. Diligenti, and C. Guardasoni, A numerical study of energetic BEM-FEM applied to wave propagation in 2D multidomains, Publications de l'Institut Mathématique - Beograd, vol. 96, no. 110 , pp. 5-22, 2014.

8. A. Aimi, M. Diligenti, A. Frangi, and C. Guardasoni, Energetic BEMFEM coupling for wave propagation in 3D multidomains, Int. J. Num. Meth. Engng., vol. 97, pp. 377-394, 2014.

9. H. Antes, G. Beer, and W. Moser, Soil-structure interaction and wave propagation problems in $2 \mathrm{D}$ by a Duhamel integral based approach and the convolution quadrature method, Comput. Mech., vol. 36, no. 6, pp. 431-443, 2005.

10. A. Bachelot, L. Bounhoure, and A. Pijols, Couplage éléments finispotentiels retardés pour la diffraction électromagnétique par un obstacle hétérogène, Numer. Math., vol. 89, pp. 257-306, 2001.

11. T. Cruse and F. Rizzo, A direct formulation and numerical solution of the general transient elastodynamic problem-I, J. Math. Anal. Appl., vol. 22, pp. 244-259, 1968.

12. A. Frangi and G. Novati, On the numerical stability of time-domain 
A.Aimi et al.

elastodynamic analyses by BEM, Comput. Methods Appl. Mech. Engrg., vol. 173, pp. 403-417, 1999.

13. M. Schanz, Application of 3D boundary element formulation to wave propagation in poroelastic solids, Eng. Anal. Boundary Elem., vol. 25, pp. 363-376, 2001.

14. A. Münch and A. F. Pazoto, Uniform stabilization of a viscous numerical approximation for a locally damped wave equation, ESAIM Control Optim. Calc. Var., vol. 13, no. 2, pp. 265-293, 2007.

15. L. R. Tcheugoué Tébou and E. Zuazua, Uniform exponential long time decay for the space semi-discretization of a locally damped wave equation via an artificial numerical viscosity, Numer. Math., vol. 95, no. 3, pp. 563-598, 2003.

16. E. Zuazua, Propagation, observation, and control of waves approximated by finite difference methods, SIAM Rev., vol. 47, no. 2, pp. 197$243,2005$.

17. A. Aimi, M. Diligenti, and C. Guardasoni, Energetic BEM-FEM coupling for the numerical solution of the damped wave equation, $A d v$. Comput. Math., pp. 1-25, 2016.

18. A. Aimi and S. Panizzi, BEM-FEM coupling for the 1D Klein-Gordon equation, Numer. Methods Partial Differential Equations, vol. 30, no. 6, pp. 2042-2082, 2014.

19. A. Bamberger and T. H. Duong, Formulation variationnelle espacetemps pour le calcul par potentiel retardé de la diffraction d'une onde acoustique. I, Math. Methods Appl. Sci., vol. 8, no. 3, pp. 405-435, 1986.

20. A. Bamberger and T. H. Duong, Formulation variationnelle pour le calcul de la diffraction d'une onde acoustique par une surface rigide, Math. Methods Appl. Sci., vol. 8, no. 4, pp. 598-608, 1986.

21. C. Lubich, Convolution quadrature and discretized operational calculus I, Numer. Math., vol. 52, pp. 129-145, 1988.

22. C. Lubich, Convolution quadrature and discretized operational calculus II, Numer. Math., vol. 52, pp. 413-425, 1988.

23. T. Ha Duong, On retarded potential boundary integral equations and their discretization, in Topics in computational wave propagation. Direct and inverse problems (P. D. et al., ed.), pp. 301-336, Springer-Verlag, 2003.

24. G. Maier, M. Diligenti, and A. Carini, A variational approach to boundary element elasto-dynamic analysis and extension to multidomain prob- 


\section{Energetic BEM ...}

lems, Comput. Methods Appl. Mech. Engrg., vol. 92, pp. 193-213, 1991.

25. A. Vick and R. West, Analysis of Damped Wave Using the Boundary Element Method, Transaction on Modelling and Simulation, vol. 15, pp. 265-278, 1997.

26. M. Costabel, Time-dependent problems with the boundary integral equation method, in Encyclopedia of Computational Mechanics (E. S. et al., ed.), pp. 1-28, John Wiley and Sons, 2004.

27. A. Aimi, M. Diligenti, and C. Guardasoni, Comparison between numerical methods applied to the damped wave equation, J. Integral Equations Appl., vol. 29, no. 1, pp. 5-40, 2017.

28. A. Aimi and M. Diligenti, A new space-time energetic formulation for wave propagation analysis in layered media by BEMs, Int. J. Numer. Meth. Engng., vol. 75, pp. 1102-1132, 2008.

29. A. Aimi, M. Diligenti, C. Guardasoni, I. Mazzieri, and S. Panizzi, An energy approach to space-time Galerkin BEM for wave propagation problems, Int. J. Numer. Meth. Engng., vol. 80, no. 9, pp. 1196-1240, 2009.

30. A. Aimi, M. Diligenti, A. Frangi, and C. Guardasoni, Neumann exterior wave propagation problems: Computational aspects of $3 \mathrm{D}$ energetic galerkin BEM, Comput. Mech., vol. 51, no. 4, pp. 475-493, 2013.

31. P. Shearer, Introduction to Seismology. Cambridge University Press, 2009.

32. J. Chevangen, N. Remacle, and X. Gallez, Discontinuous galerkin implementation of the extended helmhotz resonator impedence model in time domain, in Proceedings of the 12th AIAAA/CEAS Aeroacoustics Conference-Cambridge, AIAAA 2006-2569, 2006.

33. B. Kennett, Seismic wave propagation in stratified media. ANU-The Australian National University, 2009.

34. J. Achenbach, Wave propagation in Elastic Solids. North-Holland, 1973.

35. C. Lubich, On the multistep time discretization of linear initialboundary value problems and their boundary integral equations, $\mathrm{Nu}$ mer. Math., vol. 67, no. 3, pp. 365-389, 1994.

36. G. Monegato and L. Scuderi, Numerical integration of functions with boundary singularities, J. Comput. Appl. Math., vol. 112, no. 1-2, pp. 201-214, 1999. Numerical evaluation of integrals. 